\title{
Efecto de los revestimientos fríos sobre la temperatura superficial de las cubiertas en el clima cálido-húmedo
}

\author{
*José Alí Porras-Salazar \\ ${ }^{* *}$ Sergio Contreras-Espinoza
}

Artículo

\begin{abstract}
Resumen
Los estudios realizados en campo y laboratorio muestran que los revestimientos fríos (cool roof coatings) contribuyen a disminuir las transferencias de calor que se dan a través de las cubiertas, las cuales son una de las principales causas de malestar térmico en los climas cálidos y húmedos. No obstante, no hay suficientes investigaciones que cuantifiquen sus beneficios en el clima tropical y existe una falta de precisión técnica en gran parte del material publicitario de los productos disponibles en el mercado. Por lo tanto, con el fin de reducir esta brecha en el conocimiento, en este estudio se analizó el comportamiento térmico en campo de las láminas de hierro galvanizado con zinc antes y después de aplicarles un revestimiento frío. Como indicador se utilizó la temperatura superficial del material. Durante los dos meses que duró el experimento, se realizaron 95 observaciones a diferentes horas del día, donde se midió la temperatura superficial de la lámina con y sin revestimiento y se anotó la hora y el estado de nubosidad del cielo. Los resultandos muestran que la lámina a la que se le aplicó el revestimiento tiene una temperatura promedio $0.85^{\circ} \mathrm{C}$ más baja. Esta diferencia es estadísticamente significativa $(p=0.037)$ y es independiente de la hora del día y del estado de nubosidad del cielo. No obstante es un diferencial de temperatura menor al que encontraron los estudios previos.
\end{abstract}

Palabras clave: techos fríos; confort térmico; temperatura; tropical; pinturas reflectivas.

Cool roof coating effect on the superficial temperature of rooftops in a warm and humid weather

\footnotetext{
Abstract

Studies conducted in the field and in a laboratory show that cold roof coatings contribute to reduce heat transferability that occur through the roofs, which are one of the main causes of thermal discomfort in hot and humid climates. However, there is not enough research to quantify its benefits in tropical climates, and it lacks technical precision in many advertising material of the products available in the market. Therefore, in order to reduce this knowledge gap, this study analyzed the thermal behavior of galvanized iron sheets with zinc before and after applying a cold coating in the field. The surface temperature of the material was used as an indicator. During the two months that the experiment lasted, 95 observations were made at different times of the day where the surface temperature of the sheet with and without coating was measured, and the time and cloudiness of the sky were recorded. The results show that the sheet, in which the coating was applied, has a lower average temperature of $0.85^{\circ} \mathrm{C}$. This difference is statistically significant $(p=0.037)$ and is independent of the time of day and the cloudiness of the sky. However, the temperature differential is lower than previous studies found.
}

Keywords: cool roofs; thermal comfort; temperature; tropics; heat-reflective paint. 


\section{Introducción}

a envolvente del edificio desempeña un papel fundamental en las transferencias de calor que se producen entre los espacios exteriores e interiores (Barrios, Huelsz, Rojas, Ochoa, \& Marincic, 2012). En los edificios de poca altura, el techo representa la mayor parte de la envolvente, por lo que puede llegar a recibir más radiación solar que las paredes (Harimi et al., 2005; Jayasinghe, Attalage, \& Jayawardena, 2003; Yew et al., 2013). Esta situación se intensifica en las latitudes tropicales, donde los rayos solares inciden con mayor inclinación y en condiciones de cielo despejado los techos están sometidos a entre 6 y 8 horas de intensa radiación. La irradiancia solar puede alcanzar hasta los $1000 \mathrm{~W} / \mathrm{m}^{2}$ y entre el $20 \%$ y el $95 \%$ de esta energía es absorbida por la cubierta (Suehrcke, Peterson, \& Selby, 2008). Por lo tanto, el techo es la parte del edificio que está más expuesta al calor causado por la radiación solar (Roslan, Ibrahim, Affandi, Mohd Nawi, \& Baharun, 2016). Según el Panel Intergubernamental sobre el Cambio Climático (IPCC), en climas tropicales, el techo es responsable de aproximadamente el $30 \%$ de las ganancias de calor dentro del edificio, sin embargo, en edificios residenciales de poca altura puede llegar hasta un 70\% (Yew et al., 2013).

El exceso de calor transferido a través del techo es una de las principales causas de malestar térmico en los climas cálidos y húmedos que prevalecen en la zona tropical (Jayasinghe et al., 2003). Durante el día, la temperatura de la superficie del techo se eleva por encima de la temperatura del aire y la cubierta irradia parte de ese calor a las habitaciones de abajo, lo que aumenta la temperatura interior afectando el confort térmico de los ocupantes (Harimi et al., 2005; Roslan et al., 2016; Vecchia, Givoni, \& Silva, 2001). Por lo tanto, la selección de la forma, orientación, color y materiales del techo es de gran importancia en el rendimiento térmico de los edificios (Jayasinghe et al., 2003). Garde et al. (2004) estudiaron el efecto que varios dispositivos de protección solar tienen sobre la temperatura y el confort térmico y descubrieron que la protección solar del techo es uno de los puntos principales en el diseño térmico de edificios en el clima tropical.

En Costa Rica, la mayoría de cubiertas se construyen con láminas metálicas (Ministerio de Hacienda Costa Rica, 2017). Esto se debe, entre otros, a su durabilidad, resistencia, impermeabilidad, bajo costo, rápida instalación y casi nulo mantenimiento. Además, los techos de metal son ligeros (entre $25 \mathrm{~kg} / \mathrm{m}^{2}$ y $75 \mathrm{~kg} / \mathrm{m}^{2}$ ) si se les compara con los de tejas o concreto $\left(350 \mathrm{~kg} / \mathrm{m}^{2}\right.$ y $\left.400 \mathrm{~kg} / \mathrm{m}^{2}\right)$, por lo que la estructura que se requiere para soportarlos también es menor. No obstante, estos presentan el problema de que al ser metálicos la temperatura superficial se eleva rápidamente con la radiación solar, calor que posteriormente pasa al interior de los edificios. Los metales sin pintar suelen ser buenos reflectores solares, pero malos emisores térmicos. Un techo de metal brillante y desnudo tienen un alto factor de reflectancia solar, aproximadamente un $60 \%$, pero sus bajas emisiones térmicas, entre un 10 y $25 \%$, hace que se caliente como un techo oscuro cuando la velocidad del viento es baja (Santamouris, 2008; U.S. Environmental Protection Agency, 2008).

Sin embargo, las propiedades térmicas de las cubiertas se pueden mejorar mediante un sistema de control térmico pasivo denominado cool roofs o techos fríos, el cual consiste en aumentar la reflectancia solar y la emisión térmica de tal forma que los techos expuestos al sol tiendan a mantenerse a temperaturas más bajas que las cubiertas estándar (Akbari, Pomerantz, \& Taha, 2001; Synnefa, Santamouris, \& Akbari, 2007; Uemoto, Sato, \& John, 2010). La forma más sencilla de transformar un techo metálico ordinario en uno frío es mediante la aplicación de un recubrimiento altamente reflectivo (cool roof coating), los cuales llevan unos 25 años en el mercado mundial (Pockett \& Belusko, 2010; U.S. Environmental Protection Agency, 2008), pero fue hasta hace pocos años que comenzaron a distribuirse ampliamente en Centro América.

Este tipo de recubrimientos se pueden utilizar tanto sobre superficies existentes como nuevas, son fáciles de aplicar y el costo incremental por metro cuadrado es relativamente bajo. Según los estudios preliminares de Akbari y Desiarlais, los materiales fríos pueden costar aproximadamente EE. UU. \$1 más por metro cuadrado que los materiales convencionales y aumentar el costo total de un sistema de techo nuevo en entre $2 \%$ y $5 \%$ (2005). No obstante, existe una falta de precisión técnica 
en gran parte del material publicitario de los productos disponibles en el mercado con respecto a los beneficios térmicos (Pockett \& Belusko, 2010), lo cual se agrava en el caso Centroamericano, donde sólo se cuenta con los datos que brinda el fabricante que corresponden a condiciones de laboratorio. Por lo tanto, el objetivo de este estudio fue realizar un experimento en campo para comparar el comportamiento térmico de las láminas de hierro galvanizado con zinc antes y después de aplicar un revestimiento frío (cool roof coatings), con el fin de determinar si existe un beneficio térmico y cuál sería su dimensión. De modo similar a Uemoto, Sato y John (2010), como indicador se utilizó la temperatura de la superficie interna, que es la que estaría en contacto con el interior del edificio. Se consideró que a mayor temperatura superficial, mayor es la radiación que se irradia hacia el interior del edificio y mayor es la afectación.

Los resultados de este estudio son un insumo más para que los usuarios y todas aquellas instituciones y profesionales vinculados al ámbito de la construcción de edificios puedan realizar un análisis de costo - beneficio y comparar los resultados de esta estrategia pasiva con otras soluciones disponibles en el mercado antes de su implementación.

\section{¿Qué son los techos fríos o cool roofs?}

Los techos fríos o cool roofs son cubiertas que, incluso bajo radiación solar directa, se mantienen a temperaturas superficiales más bajas que las cubiertas convencionales al minimizar la absorción solar y maximizar la emisión térmica (Akbari \& Levinson, 2008). Un techo frío es altamente efectivo reflejando la radiación solar incidente y emitiendo energía térmica en longitudes de onda infrarrojas. El calor del sol se devuelve a la atmósfera en lugar de ser absorbido y transferido al edificio. Qué tan frío estará el techo, dependerá de su reflectancia solar, emisión térmica y la velocidad del viento (Akbari \& Levinson, 2008).

El Índice de Reflexión Solar, SRI por sus siglas en inglés, es una medida que combina los índices de reflectancia solar y emisividad térmica de los materiales. Se podría resumir como la capacidad del techo para rechazar el calor solar. De esta forma, se puede utilizar como un indicador de la probabilidad que existe de que un material se caliente cuando recibe radiación solar. EI SRI se mide en una escala de 0 a 100, donde un techo de color negro normal $(0,05$ reflectancia, emitancia 0,90$)$ tiene un valor de 0 y uno de color blanco (0,80 reflectancia, emitancia 0,90$)$, un valor de 100 . Por lo tanto, las superficies que absorben y retienen la radiación solar y, por lo tanto, se calientan con la luz del sol, tienen un número más bajo, mientras que los materiales altamente reflectantes y emisivos tienen un número mayor. Cuanto más alto sea el valor de SRI, más baja será la temperatura de la superficie expuesta a la radiación.

El sistema estadounidense de certificación de edificios sostenibles LEED del Consejo de la Construcción Verde de Estados Unidos otorga el Crédito 7.2, Efecto isla de calor de la Categoría 1, Sitios sostenibles, a aquellos edificios que tengan techos fríos. Para obtener dicho crédito, las cubiertas con una pendiente $\leq 15 \%$ deben de tener un SRI $\geq$ 78 , mientras que en aquellas que presentan una pendiente $>15 \%$, el SRI debe ser $\geq$ 29. En términos generales, casi cualquier tipo de cubierta puede ser un techo frío, ya que es la superficie expuesta al sol la que determina si es frío o no (U.S. Department of Energy, 2019). Sin embargo, dado que el tipo de superficie de techo expuesta cambia con la pendiente, los productos para techos fríos se clasifican en los que se pueden aplicar en cubiertas de baja inclinación y los que se utilizan en cubiertas con pendientes pronunciadas (U.S. Environmental Protection Agency, 2008).

El techo frío ha venido ganando popularidad como una solución térmica pasiva (Zingre et al., 2015) porque permite el ahorro de energía al disminuir las necesidades de refrigeración en los edificios, mejora el ambiente térmico de aquellos espacios que no tienen aire acondicionado, disminuye la temperatura de los techos lo cual puede extender su vida útil y reduce el efecto de isla de calor (U.S. Department of Energy, 2019; Akbari and Levinson, 2008). Investigaciones previas han indicado que los ahorros son mayores para los edificios ubicados en climas con largas temporadas de enfriamiento y cortas temporadas de calefacción (Konopacki, Gartland, Akbari, \& Rainer, 1998; Santamouris, 2008), lo cual los hace ideales para los climas cálidos y húmedos. 
La forma más sencilla de transformar un techo ordinario en uno frío es mediante la aplicación de un recubrimiento altamente reflectivo (cool roof coating). Estos revestimientos suelen ser pinturas gruesas, blancas o con pigmentos especiales altamente reflectantes (U.S. Department of Energy, 2019). Algunas proporcionan la misma gama de colores visibles que los productos estándar, pero funcionan de manera diferente en la región infrarroja del espectro, reflejando más de la parte invisible del espectro solar. (Pockett \& Belusko, 2010). Se pueden aplicar tanto sobre techos con pendientes leves como pronunciadas y sobre una amplia gama de superficies, incluyendo capas de asfalto, grava, metal e incluso sobre membranas de una sola capa. De acuerdo con la EPA, existen tres tipos de revestimientos: (1) los cementicios que contienen partículas de ese material, (2) los elastoméricos que contienen polímeros y (3) los que contienen tanto partículas cementicias como polímeros (U.S. Environmental Protection Agency, 2008). En la actualidad, existen revestimientos con una reflectancia y emitancia térmica de hasta de un 90\% (U.S. Environmental Protection Agency, 2019) y que además pueden proteger la superficie del techo de la luz ultravioleta (UV).

\section{Metodología}

\section{Materiales}

Mediante búsquedas por internet y llamadas telefónicas a los principales fabricantes y distribuidores de pinturas y recubrimientos de Costa Rica, se localizaron aquellos productos que en su publicidad manifestaran mejorar las condiciones térmicas del techo, ya sea por sus propiedades altamente reflectantes o de aislamiento térmico (heatreflective paint o termal insulation paint). La pintura podía ser de producción nacional o importada, pero con amplia distribución en el país. En el caso de las importadas, se solicitó que tuvieran la misma disponibilidad que las nacionales, con el fin de evitar las pinturas que son importadas para proyectos inmobiliarios específicos, ya que no están disponibles para la mayoría de los consumidores.

Se encontró un único recubrimiento disponible, que es una pintura a base de agua con acabado color blanco mate. Tanto el envase como la ficha técnica indican que funciona como aislante térmico y cuenta con propiedades que ayudan a reducir la temperatura. El recipiente indica además que proporciona aislación a los rayos infrarrojos. No fue posible obtener los componentes de la pintura, ni información sobre la tecnología utilizada para mejorar las propiedades térmicas. La publicidad y ficha técnica tampoco brinda datos sobre la reflectancia, emitancia térmica o el Índice de Reflexión Solar (SRI). La pintura tampoco se encuentra en la lista de productos de techos certificados por el programa Energy Star, de la Agencia de Protección Ambiental de los EE.UU. (EPA por sus siglas en inglés) (U.S. Environmental Protection Agency, 2019). En la Tabla 1 se muestran las propiedades físicas de la pintura de acuerdo a la ficha técnica proporcionada por el fabricante.

Tabla 1. Propiedades físicas del recubrimiento utilizado según la ficha técnica del fabricante. Nota del fabricante: Datos calculados bajo condiciones de laboratorio.

\begin{tabular}{l|l} 
Propiedades físicas: & Datos: \\
\hline Sólidos por volumen (\%) & $67-69$ \\
\hline Sólidos por peso (\%) & $52-54$ \\
\hline Peso por galón (kg/gal) & $2.8-3.0$ \\
\hline Viscosidad Stormer Krebs (Ku) & $110-120$ \\
\hline
\end{tabular}

\section{Configuración del experimento}

El estudio se realizó en un predio ubicado en Tilarán, Guanacaste $\left(10.47^{\circ} \mathrm{N}, 84.96^{\circ}\right.$ 0) a 550 metros sobre el nivel del mar. El sitio estaba cubierto de césped y no tenía construcciones 0 árboles que pudieran servir de obstáculos a la radiación directa durante el periodo de estudio, de marzo a mayo entre las 8 a.m. y las 5 p.m (Ver Figura 1). Se decidió realizar un estudio de campo ya que según Pockett y Belusko (2010) las lámparas infrarrojas utilizadas en los estudios desarrollados en laboratorios sobreestiman los beneficios de las pinturas que reflejan el calor. 


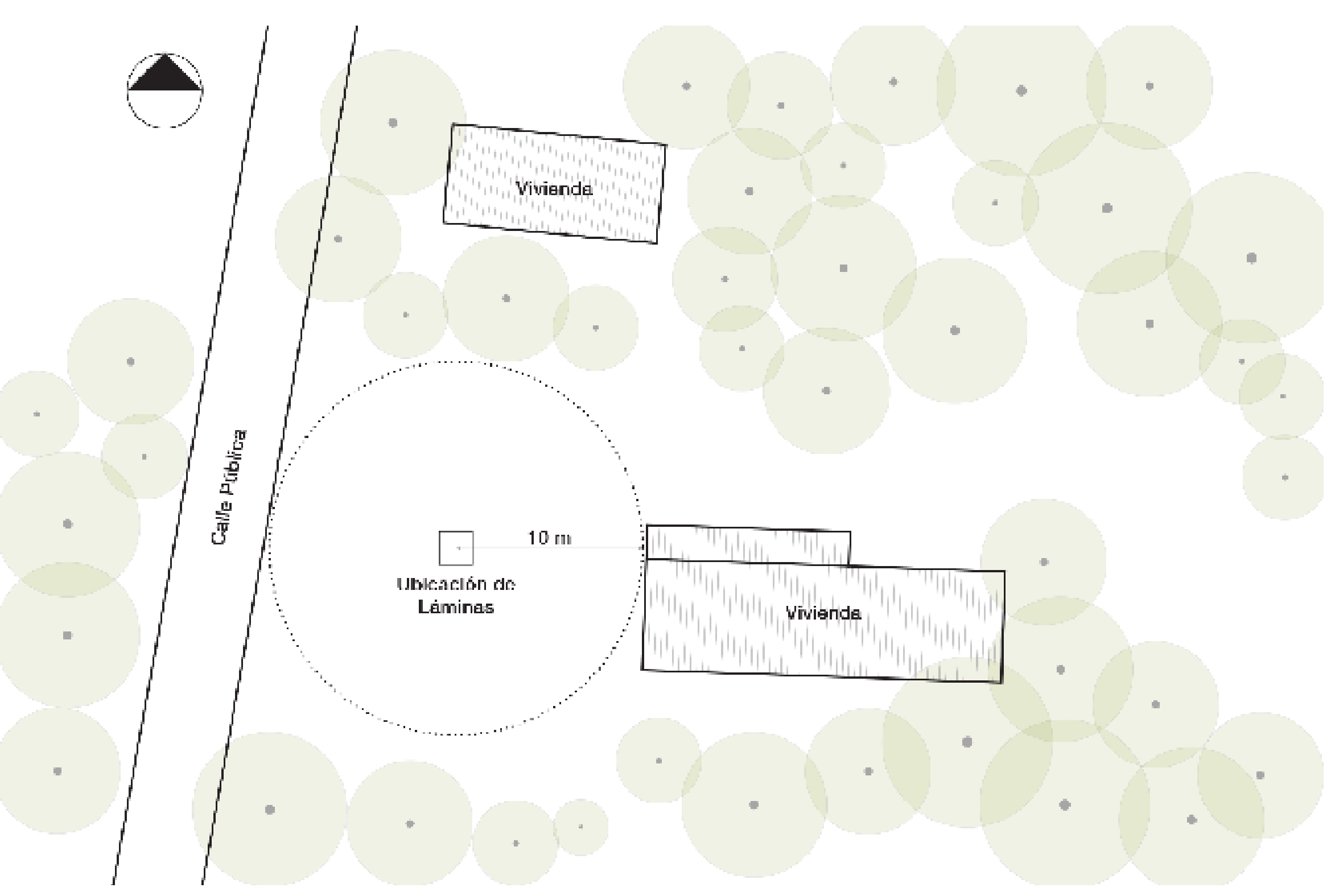

Figura 1. Planta de conjunto del sitio donde se ubicaron las láminas de hierro galvanizado con zinc con las que se realizó el experimento. El sitio está libre de obstáculos que pudieran generar algún tipo de sombreamiento sobre las láminas durante el periodo de estudio.

Fuente: Elaboración propia.

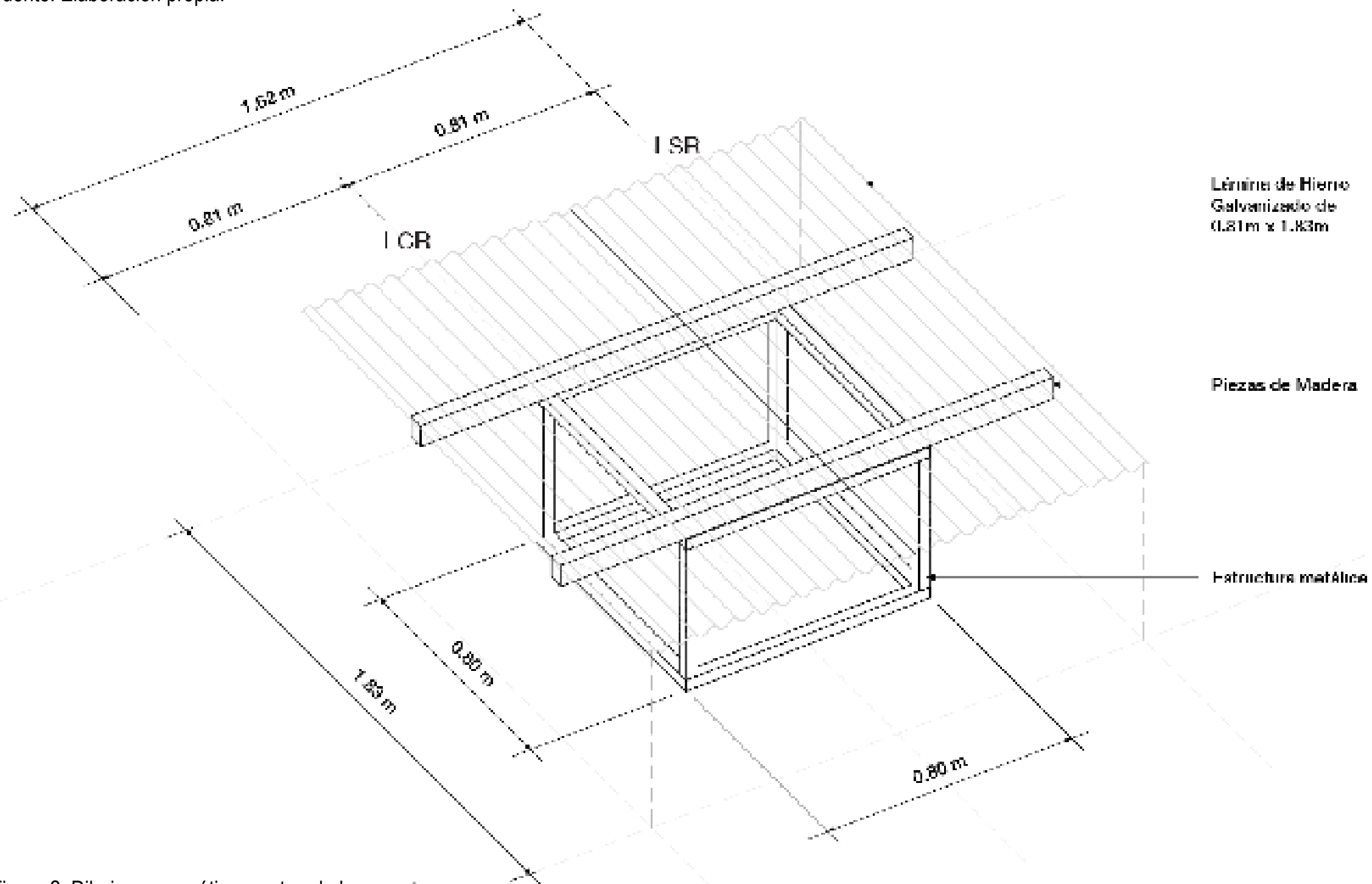


Como se muestra en la Figura 2, dos láminas nuevas de hierro galvanizado de $81 \mathrm{~cm}$ de ancho por $183 \mathrm{~cm}$ de largo y 0.38 milímetros de espesor se instalaron, una a la par de la otra, sobre una base de metal y madera a $50 \mathrm{~cm}$ del terreno. Siguiendo las especificaciones del fabricante, se eliminó toda la suciedad de las láminas y se aplicó el recubrimiento en un día sin lluvia y de baja humedad (65\%). La aplicación se llevó a cabo aproximadamente a las 10 a.m.

Una de las láminas (LCR) se cubrió con 2 capas de pintura para alcanzar el espesor recomendado por el fabricante de aproximadamente $0.32 \mathrm{~mm}(\approx 15$ mils). Se consideró un espesor por capa de $0.2 \mathrm{~mm}$ o superior ( $\approx 7.5$ mils). La aplicación se llevó a cabo con una brocha. Dado que el producto viene listo para usar, se aplicó directamente. La otra lámina se dejó desnuda (LSR). Con el fin de que las condiciones del experimento se acercaran lo más posible a la realidad, durante el periodo de toma de datos no se realizó ningún tipo de limpieza en la superficie de las dos láminas.

\section{Mediciones}

La medición de la temperatura superficial de las láminas se realizó entre el 14 de marzo y el 15 de mayo en diferentes momentos del día, pero no antes de las 8 a.m. ni después de las 5 p.m. Se utilizó un termómetro infrarrojo de la marca Extech, modelo 42529 con una precisión de $\pm 2 \%$. Este dispositivo fue calibrado en fábrica y se usó dentro de los 12 meses posteriores a la compra. El termómetro infrarrojo se utilizó según las especificaciones del fabricante. La medición se realizó en el centro de la lámina a una distancia no mayor a los $15 \mathrm{~cm}$. La temperatura del aire y la humedad relativa se monitorearon con un Hobo U12-12 con una precisión de $\pm 0.35^{\circ} \mathrm{C}$ y $\pm 2.5 \%$ respectivamente, ubicado a $15 \mathrm{~m}$ del sitio donde se colocaron las láminas.

Además de la temperatura superficial de las láminas, en cada observación, el experimentador anotó en la hoja de control el estado del cielo según la cobertura de nubosidad. Se utilizó D para despejado, SN para seminublado, N para nublado y NLL para nublado con lluvia. Para realizar la clasificación, se utilizaron los siguientes criterios: (1) Despejado: se observa una cobertura de nubosidad menor a un $20 \%$ de la bóveda celeste visible, (2) Seminublado: se observa una cobertura de nubosidad mayor a un $20 \%$ pero menor a un $70 \%$ de la bóveda celeste visible, (3) Nublado: se observa una cobertura de nubosidad mayor a un $70 \%$ de la bóveda celeste visible y (4) Nublado con lluvia: se observa una cobertura de nubosidad mayor a un $70 \%$ de la bóveda celeste visible y hay precipitación en el sitio donde se encuentran las láminas.

\section{Tratamiento estadístico de los datos}

Para evaluar el efecto del recubrimiento frío en la temperatura superficial de las láminas, se realizó un análisis estadístico usando el software $R$ ( $R$ Core Team, 2016). Se llevó a cabo una comparación de las medidas de las dos muestras de datos mediante un t-test. Debido a que estudios anteriores en campo y laboratorio (Dabaieh, Wanas, Amer, \& Johansson, 2015; Shen, Tan, \& Tzempelikos, 2011; Synnefa et al., 2007; Uemoto et al., 2010) han sugerido que después de aplicar un recubrimiento frío la temperatura superficial de los techos tiende a bajar, la prueba de hipótesis se formuló esperando esta disminución de temperatura ( $\mathrm{H} 0: \mu 1<\mu 2$ versus $\mathrm{H} 1: \mu 1<\mu 2)$, donde $\mu 1$ representa la temperatura promedio de la lámina con recubrimiento y $\mu 2$ la temperatura promedio de la lámina sin recubrimiento.

Posteriormente, se analizó el efecto del recubrimiento frío sobre las láminas de hierro galvanizado, controlando la influencia de la hora del día. Para ello, se utilizó un modelo de regresión lineal en el cual la temperatura superficial es la variable dependiente y la hora del día y una variable dicótoma (dummy), que indica la presencia o no del recubrimiento, son las variables explicativas. Se consideró como significativa una estimación, esto es diferente de cero, si su valor-p fue menor que 0.05 .

Con la finalidad de complementar las pruebas anteriores y determinar el efecto conjunto del recubrimiento y el tipo de cielo, se realizó un análisis de varianza (ANOVA) de dos factores. Ante la probabilidad de que el ANOVA rechazara la Hipótesis nula de igualdad 
de medidas de las muestras, se planteó utilizar el test de comparaciones múltiples LSD

(Least significant difference), con el fin de determinar cuáles pares de medidas son diferentes, ya que el rechazo de la Hipótesis nula puede surgir de cualquiera de los pares de observados.

\section{Resultados}

\begin{tabular}{c|c|c|c|c|c|c}
\multirow{2}{*}{ Hora } & \multirow{2}{*}{$\begin{array}{c}\text { Número de } \\
\text { observaciones }\end{array}$} & Temperatura superficial $\left({ }^{\circ} \mathrm{C}\right)$ & \multicolumn{2}{|c|}{ Temperatura superficial $\left({ }^{\circ} \mathrm{C}\right)$} & \multirow{2}{*}{ Diferencia entre las medidas } \\
\cline { 3 - 6 } & LCR & SD & LSR & SDCR) \\
\hline $8: 00$ & 24 & 28.3 & 2.7 & 29.1 & 2.8 & 0.8 \\
\hline 9:00 & 8 & 31.0 & 1.6 & 32.1 & 1.2 & 1.1 \\
\hline $10: 00$ & 4 & 31.3 & 1.5 & 32.3 & 1.9 & 1.0 \\
\hline $11: 00$ & 1 & - & - & - & - & - \\
\hline $12: 00$ & 34 & 33.3 & 2.6 & 34.1 & 2.6 & 0.8 \\
\hline $13: 00$ & 4 & 33.0 & 1.4 & 34.5 & 1.3 & 1.5 \\
\hline $14: 00$ & 2 & 33.5 & 0.7 & 35.0 & 1.4 & 1.5 \\
\hline $16: 00$ & 18 & 29.4 & 2.7 & 30.0 & 2.8 & 0.6 \\
\hline
\end{tabular}

SD: Desviación estándar

Tabla 2. Caracterización de observaciones por hora.
A lo largo de los dos meses que duró el experimento, se realizaron 95 observaciones. Treinta y seis en la mañana, 34 al medio día y 25 en la tarde. El número de observaciones realizadas por hora se presentan en la Tabla 2, así como la temperatura superficial promedio con y sin recubrimiento y la desviación estándar por hora. Se puede observar que la temperatura media siempre fue mayor en la lámina sin recubrimiento (LSR) y que la diferencia se mantuvo entre $0.6^{\circ} \mathrm{C}$ y $1.5^{\circ} \mathrm{C}$.

Sesenta y cinco de los registros corresponden a cielo despejado (68.4\%), 17 a seminublado (17.9\%), 9 a nublado $(9.5 \%)$ y solo 2 a nublado con lluvia $(2.1 \%)$. Para dos de los datos, el experimentador no anotó el tipo de cielo. El número de resultados por tipo de cielo guarda relación con la época en que se realizaron las mediciones, que se caracteriza por ser una estación de cielos despejados y pocas lluvias. El número de tomas realizadas por tipo de cielo se presenta en la Tabla 3, así como la temperatura superficial promedio con y sin recubrimiento y la desviación estándar por hora. Nuevamente la temperatura media de la lámina sin recubrimiento (LSR) fue mayor sin importar el tipo de cielo. La temperatura media de la lámina desnuda, considerando todas las horas y tipos de cielo, fue de $31.9^{\circ} \mathrm{C}\left(\mathrm{SD} 3.3^{\circ} \mathrm{C}\right)$, mientras que la de la lámina recubierta fue de $31.0^{\circ} \mathrm{C}\left(\mathrm{SD} 3.3^{\circ} \mathrm{C}\right)$. La diferencia entre ambas medidas, $0.85^{\circ} \mathrm{C}(\mathrm{DE}$ $\left.0.9^{\circ} \mathrm{C}\right)$, es estadísticamente significativa $(p=0.037)$.

\begin{tabular}{c|c|c|c|c|c|c}
\multirow{2}{*}{ Hora } & \multirow{2}{*}{$\begin{array}{c}\text { Número de } \\
\text { observaciones }\end{array}$} & \multicolumn{2}{|c|}{ Temperatura superficial $\left({ }^{\circ} \mathrm{C}\right)$} & Temperatura superficial $\left({ }^{\circ} \mathrm{C}\right)$ & Diferencia entre las medidas \\
\cline { 3 - 6 } & & LCR & SD & LSR & SD & 0.9 \\
\hline Despejado & 65 & 31.9 & 3.0 & 32.8 & 3.0 & 0.7 \\
\hline Seminublado & 17 & 28.1 & 3.5 & 28.8 & 3.5 & 0 \\
\hline Nublado & 9 & 24.5 & 2.1 & 24.5 & 2.1 & 1.0 \\
\hline $\begin{array}{c}\text { Nublado con } \\
\text { Iluvia }\end{array}$ & 2 & 29.9 & 2.3 & 30.9 & 2.5 & 0 \\
\hline
\end{tabular}

\section{SD: Desviación estándar}

Tabla 3. Caracterización de las observaciones por tipo de cielo.
Los resultados de la regresión lineal con la variable dicótoma (dummy) y controlando el efecto de la hora del día en que se realizó la observación muestran que la temperatura superficial promedio de la lámina sin pintar es $0.85^{\circ} \mathrm{C}$ más alta que la de la lámina pintada. Este resultado es significativo $(p<0.001)$.

En los resultados del ANOVA que se muestran en la Tabla 4, se puede observar que no existe interacción entre el recubrimiento aplicado y el tipo de cielo $(p=0.9813)$. Esto se ve reflejado en la Figura 3, donde las medidas de las temperaturas superficiales de los diferentes niveles de los factores analizados no se cruzan en ningún momento. A partir del ANOVA, se pudo observar que la temperatura superficial de la lámina es afectada tanto por el tratamiento $(p=0.0443)$, lo que guarda relación con los resultados del t-test expuestos arriba, pero también es afectada por el tipo de cielo ( $p=1.31$ e 7). 
Tabla 4. Resultados del ANOVA

De acuerdo con los resultados del test de la diferencia menos significativa (LSD) (Tabla 5), todos los pares de medidas correspondientes al tipo de cielo son significativamente distintos. En ninguno de estos análisis se incluyeron las observaciones de los días nublados con lluvia (NLL) ya que solo se contaba con dos datos.

\begin{tabular}{|c|c|c|c|c|c|}
\hline Efecto & $\begin{array}{c}\text { Grados de } \\
\text { libertad }\end{array}$ & $\begin{array}{l}\text { Suma de } \\
\text { Cuadrados }\end{array}$ & $\begin{array}{l}\text { Cuadrados } \\
\text { Medios }\end{array}$ & $\mathbf{F}$ & Valor-p \\
\hline \multicolumn{6}{|c|}{ Con el efecto de la interación } \\
\hline Tipo de Cielo & 1 & 35.2 & 35.2 & 4,095 & $0.0455^{*}$ \\
\hline Cielo & 2 & 297.3 & 148.63 & 17.056 & $1.56 \mathrm{e}-07^{*}$ \\
\hline Interacción & 2 & 0.3 & 0.16 & 0.019 & 0.9813 \\
\hline Residuos & 176 & 1524.8 & 8.66 & & \\
\hline \multicolumn{6}{|c|}{ Sacando el efecto de la interacción } \\
\hline Tipo de Cielo & 1 & 35.2 & 35.2 & 4,104 & $0.0443^{*}$ \\
\hline Cielo & 2 & 297.3 & 148.63 & 17.348 & $1.31 \mathrm{e}-07^{*}$ \\
\hline Residuos & 178 & 1525.1 & 8.57 & & \\
\hline
\end{tabular}

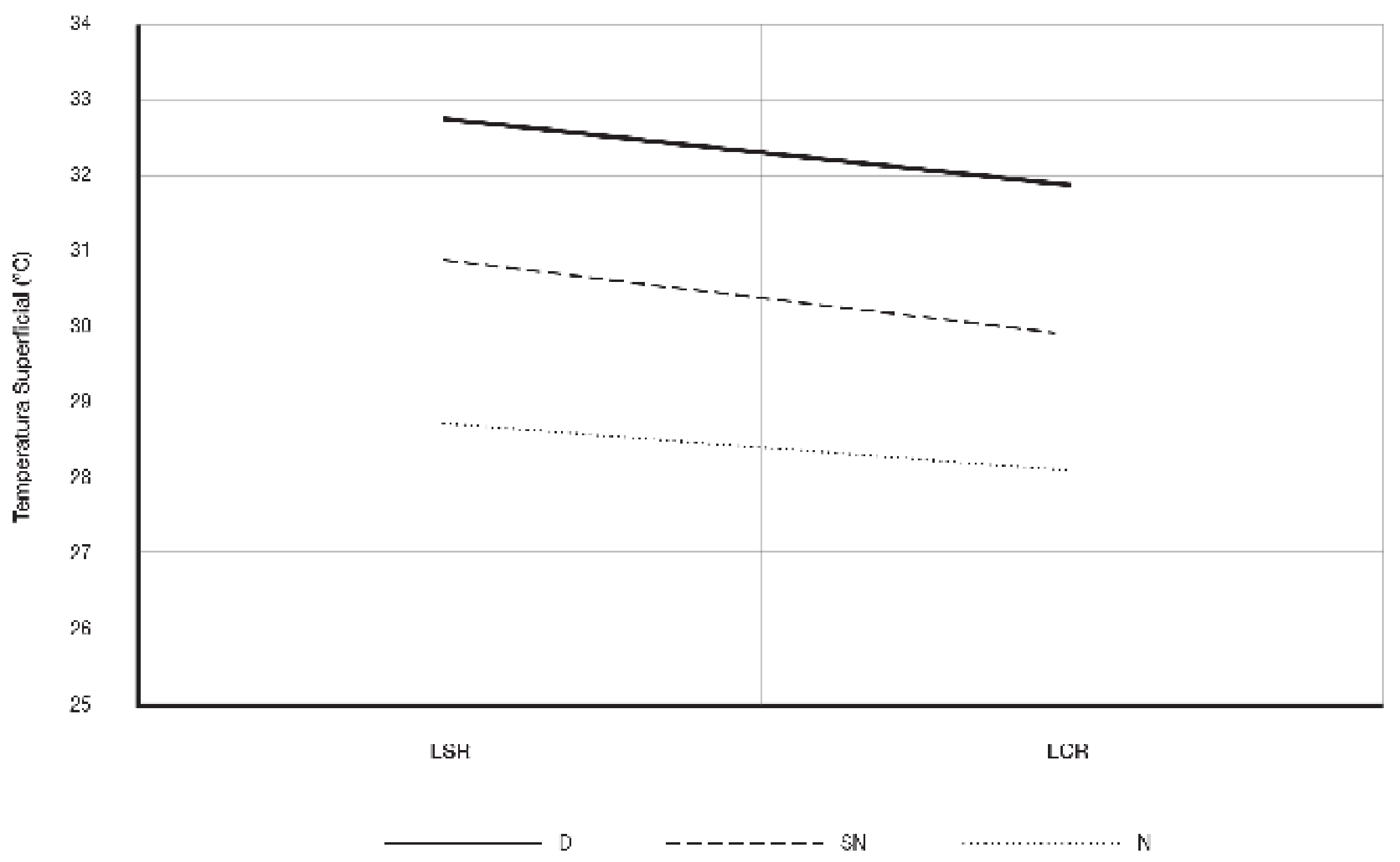

Figura 3. Temperatura superficial promedio de la $A$ lámina sin recubrimiento (LSR) y la lámina con recubrimiento (LCR) según el estado de nubosidad del cielo. No se presenta el estado de cielo Nublado con lluvia (NLL) porque solo se cuenta con dos datos. Fuente: Elaboración propia. 


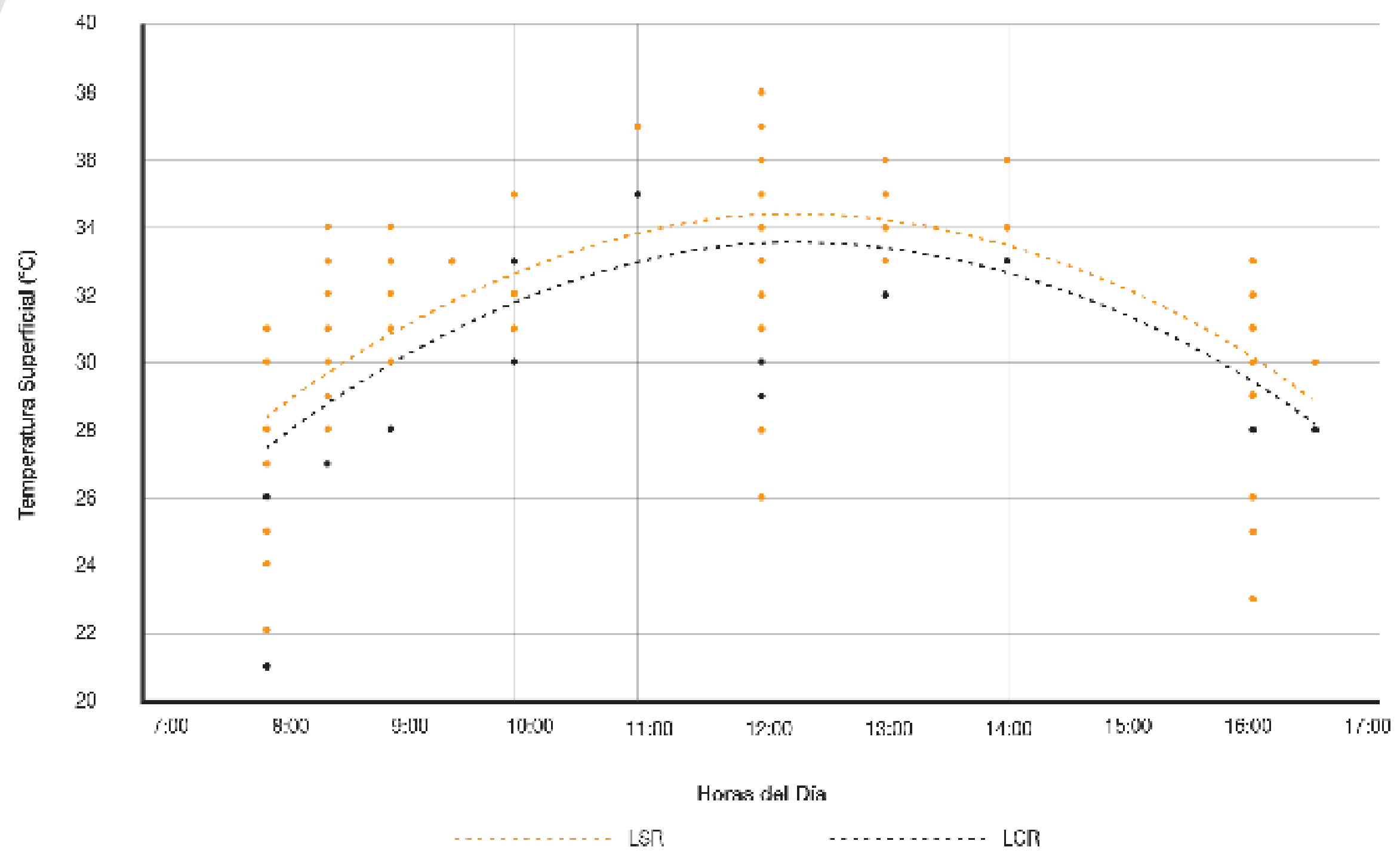

Figura 4. Las temperaturas superficiales $(T)$ de la lámina sin y con recubrimiento se plotearon frente a las horas de un día tipo (8 a.m. a 5 p.m.). Las líneas representan los polinomios cuadráticos ajustados, mientras que los puntos indican cada una de las observaciones realizadas. La función que describe la relación entre la temperatura superficial de la lámina desnuda y la hora del día es la siguiente: $y=-0.321 \mathrm{~T}^{2}+7.914 \mathrm{~T}-14.305 . \mathrm{R}^{2}$ $=0.479 ; p<0.001$. Por su parte la función que describe la relación entre la temperatura superficial de la lámina recubierta es la siguiente: $y=-0.314 \mathrm{~T}^{2}$ $+7.761 \mathrm{~T}-14.446 . \mathrm{R}^{2}=0.486 ; p<0.001$.

Fuente: Elaboración propia.

Tabla 5. Resultados del método de la diferencia menos significativa (LSD). Letras iguales implica medias iguales, por lo tanto, como las letras de cada grupo son diferentes, entonces todas las medias también son diferentes.
Finalmente, la temperatura media del aire cuando se realizaron las mediciones sobre las láminas fue de $27.7^{\circ} \mathrm{C}$., es decir, $4.2^{\circ} \mathrm{C}$ menos que la lámina desnuda y $3.3^{\circ} \mathrm{C}$ menos que la recubierta. Sin embargo, durante el periodo de mediciones, se observaron diferencias pico de hasta $8.7^{\circ} \mathrm{C}$ menos con respecto a la lámina desnuda y $6.9^{\circ} \mathrm{C}$ menos con respecto a la lámina con tratamiento.

\begin{tabular}{c|c|c}
$\begin{array}{c}\text { Cobertura de cielo según estado } \\
\text { de nubosidad }\end{array}$ & Temperatura media $\left({ }^{\circ} \mathrm{C}\right)$ & Grupo \\
\hline Despejado (D) & 32.4 & $\mathrm{~A}$ \\
\hline Seminublado (SN) & 30.4 & $\mathrm{~B}$ \\
\hline Nublado (N) & 28.4 & $\mathrm{C}$ \\
\hline
\end{tabular}

\section{Discusión}

Los resultados del experimento muestran que la temperatura superficial media de la lámina de hierro galvanizado a la que se le aplicó un recubrimiento frío (LCR) es de $0.85^{\circ} \mathrm{C}$ más baja que la de la lámina que se dejó desnuda (LSR). Esta diferencia es estadísticamente significativa ( $p=0.037$ ) y, como se puede observar en la Figura 4 , se mantiene prácticamente uniforme a lo largo del día. Estos resultados guardan relación con los hallazgos encontrados en estudios previos realizados tanto en el campo como en el laboratorio, cuando a una cubierta expuesta a la radiación solar se le aplica un recubrimiento frío que aumenta sus índices de reflexión y emisividad, su temperatura superficial tiende a bajar. No obstante el diferencial de temperatura encontrado en este experimento fue mucho menor.

Yew et al. (2013) realizaron un estudio en laboratorio donde compararon la temperatura superficial de una lámina de hierro galvanizado cubierta con una pintura convencional contra las de una lámina con una "pintura térmica aislante". Los resultados muestran que la diferencia entre ambas fue de $4{ }^{\circ} \mathrm{C}$. Uemoto, Sato y John (2010) expusieron láminas de fibrocemento con diferentes recubrimientos y colores a la radiación infrarroja en un laboratorio. Se encontró que, para láminas del mismo color, las temperaturas superficiales de las láminas con recubrimientos fríos fueron más de $10^{\circ} \mathrm{C}$ más bajas que las de las láminas con pinturas convencionales. La menor diferencia de temperatura 
se presentó entre los acabados "blanco frío" y "blanco convencional", $5.8^{\circ} \mathrm{C}$, mientras que la mayor se dio entre "blanco frío" y "café convencional" que fue de $23.1^{\circ} \mathrm{C}$. Por su parte Synnefa, Santamouris y Livada (2006) demostraron que los recubrimientos reflectantes pueden reducir la temperatura superficial de las baldosas de concreto blanco en hasta $4{ }^{\circ} \mathrm{C}$ en condiciones de verano.

Una de las razones que podrían explicar la diferencia de temperatura entre las dos láminas no fue mayor es porque los dos materiales son altamente reflexivos y algunos estudios muestran comparaciones realizadas entre techos de colores oscuros con baja reflexión y baja emisividad (U.S. Environmental Protection Agency, 2008), como los de teja asfáltica color negro con techos a los que se les ha aplicado un recubrimiento frío (Uemoto et al., 2010). En este caso, se debe tomar en cuenta que es posible que con el tiempo la lámina desnuda vaya perdiendo parte de estas propiedades y suba la temperatura, no obstante, esto es una mera especulación que tendrá que comprobarse en futuros experimentos. Otra explicación podría estar relacionada con el acabado de la pintura. El producto utilizado es mate y se aplicó con brocha siguiendo las recomendaciones del fabricante, pero lo que se recomienda es emplear una pintura brillante y rociarla para obtener una superficie lo más lisa posible que no capture rápidamente la suciedad y el polvo (Pockett \& Belusko, 2010).

La nubosidad del cielo afecta la temperatura superficial de las láminas. Las mayores temperaturas se dan con los cielos despejados, mientras que las menores con los cielos nublados. Esto tiene lógica ya que las nubes obstaculizan parte de la radiación solar y, por lo tanto, es menos el calor que llega hasta las láminas. Sin embargo, la Tabla 3 y la Figura 3 muestran que sin importar el tipo de cielo la temperatura de la lámina con recubrimiento (LCR) siempre será menor. Esta diferencia parece disminuir conforme aumenta la nubosidad, no obstante, se debe tomar en cuenta que los datos de seminublado y nublado, por la época del año en que se realizó el estudio son pocos. Nuevos experimentos con un grupo de datos más balanceado serían necesarios para ver si esta tendencia se mantiene.

En el presente estudio, no fue posible determinar si la disminución en la temperatura superficial de la lámina LCR se debió a que, con la aplicación del recubrimiento, se mejoró el índice de reflectancia solar, o el índice de emisividad, o ambos. Lamentablemente no se pudieron hacer análisis de laboratorio para estimarlos y la información no está especificada en la hoja técnica del producto. Esto es algo que será necesario tomar en cuenta en futuros estudios. En futuros experimentos también se tendrá que analizar si con solo aumentar la reflectancia solar, pintando la lámina de blanco brillante se logran resultados similares. Otra limitación del estudio es que solo hubiera disponible en el mercado un recubrimiento frío (cool roof coating), aun así no se quisieron incluir otros productos que, aunque en otros contextos puedan haber demostrado ser eficientes, no se distribuyen en la región y por lo tanto no están al alcance del consumidor promedio.

En el experimento, se utilizó como indicador del comportamiento térmico de las láminas la temperatura superficial de la parte de debajo de la lámina, por ser la que estaría en contacto con el interior del edificio. Este mismo indicador fue utilizado en su investigación por Uemoto, Sato y John (2010). Además, mediciones puntuales realizadas a lo largo de los dos meses demostraron que la diferencia entre ambas caras no fue lo suficientemente grande como para generar cambios en los registros tomados por el termómetro infrarrojo utilizado.

Los resultados de este estudio se pueden utilizar para hacer estimaciones sobre la temperatura interior de los aposentos cuyos techos o paredes estén pintados con un recubrimiento frío o para estimar posibles reducciones en el consumo de energía en los edificios acondicionados mecánicamente. No obstante, se debe tomar en cuenta que en dichos cálculos la temperatura superficial de la cubierta tan solo será una entre las muchas variables que deberán ser consideradas. Es por ello que las afirmaciones publicitarias de los fabricantes en las que se dice que su pintura reducirá la temperatura ambiente en un número determinado de grados deben tomarse con cautela (Pockett \& Belusko, 2010). La misma reserva merecen afirmaciones como que el revestimiento tiene propiedades de aislamiento, ya que reducir la transmisión de calor no representa una parte importante de los beneficios que este tipo de recubrimientos aportan. 
Los resultados aquí presentados son un insumo para llevar a cabo análisis de costobeneficio cuando se consideren utilizar revestimientos fríos en las cubiertas de los edificios. No obstante, los datos deberán ser validados en estudios futuros y en otros contextos diferentes al utilizado en este experimento.

\section{Conclusiones}

Se llevó a cabo un estudio de campo en un clima cálido-húmedo para analizar los efectos de los revestimientos fríos (cool roof coatings) sobre la temperatura superficial de las láminas de hierro galvanizado con zinc, que es el tipo de cubierta más utilizado en Costa Rica. Los resultados del estudio muestran que la temperatura superficial media de la lámina de hierro a la que se le aplicó un recubrimiento frío (LCR) es en promedio, $0.85^{\circ} \mathrm{C}$ más baja que la de la lámina que se dejó desnuda (LSR). La diferencia entre ambas medidas es estadísticamente significativa $(p=0.037)$. Esta diferencia se mantuvo independiente de la hora del día y del estado de nubosidad del cielo. Aun cuando la aplicación del revestimiento brinda efectos térmicos positivos, ya que al disminuir la temperatura superficial de la lámina también disminuye la radiación que esta irradiaría hacia el interior de los edificios, los beneficios encontrados son menores a los que se hallaron en estudios previos. Lamentablemente esta investigación no logró determinar si la disminución en la temperatura superficial de la lámina LCR se debió a la aplicación del recubrimiento y si esta mejoró el índice de reflectancia solar, o el índice de emisividad, 0 ambos.

\section{Referencias bibliográficas}

Akbari, H \& Desjarlais, A. (2005). Cooling down the house: residential roofing products soon will boast "cool surfaces". Professional Roofing, March, 32-38. Recuperado de http://www.professionalroofing.net/article.aspx?_ID=609.

Akbari, H., \& Levinson, R. (2008). Evolution of cool-roof standards in the US. Advances in Building Energy Research, 2(1), 1-32. http://doi.org/10.3763/aber.2008.0201

Akbari, H., Pomerantz, M., \& Taha, H. (2001). Cool surfaces and shade trees to reduce energy use and improve air quality in urban areas. Solar Energy, 70(3), 295-310. Recuperado de http://www.sciencedirect.com/science/article/pii/ S0038092X0000089X

Barrios, G., Huelsz, G., Rojas, J., Ochoa, J. M., \& Marincic, I. (2012). Envelope wall/roof thermal performance parameters for non air-conditioned buildings. Energy and Buildings, 50, 120-127. http://doi.org/10.1016/j.enbuild.2012.03.030

Dabaieh, M., Wanas, O., Amer, M., \& Johansson, E. (2015). Reducing cooling demands in a hot dry climate : A simulation study for non-insulated passive cool roof thermal performance in residential buildings. Energy \& Buildings, 89, 142-152. http://doi. org/10.1016/j.enbuild.2014.12.034

Garde, F., Adelard, L., Boyer, H., \& Rat, C. (2004). Implementation and experimental survey of passive design specifications used in new low-cost housing under tropical climates. Energy and Buildings, 36(4), 353-366. http://doi.org/10.1016/j. enbuild.2004.01.045

Harimi, D., Harimi, M., Kurian, V. J., Bolong, N., Zakaria, I., \& Gungat, L. (2005). Evaluation of the Thermal Performance of Concrete Tile under Tropical Climatic Conditions. In Conference on Sustainable Building South East Asia (p. 13). Malaysia.

Jayasinghe, M. T. R., Attalage, R. A., \& Jayawardena, A. I. (2003). Roof orientation, roofing materials and roof surface colour: their influence on indoor thermal comfort in warm humid climates. Energy for Sustainable Development, 7(1), 16-27. http:// doi.org/10.1016/S0973-0826(08)60345-2

Konopacki, S., Gartland, L., Akbari, H., \& Rainer, L. (1998). Demonstration of Energy Savings of Cool Roofs. California, USA: Berkeley National Laboratory 
Pockett, J., \& Belusko, M. (2010). A Review of Heat-reflective Paints. In 48th AuSES Annual Conference. Canberra.

R Core Team. (2016). R: A Language and Environment for Statistical Computing. Vienna, Austria: R Foundation for Statistical Computing. Recuperado de https:// www.r-project.org/

Roslan, Q., Ibrahim, S. H., Affandi, R., Mohd Nawi, M. N., \& Baharun, A. (2016). A literature review on the improvement strategies of passive design for the roofing system of the modern house in a hot and humid climate region. Frontiers of Architectural Research, 5(1), 126-133. http://doi.org/10.1016/j.foar.2015.10.002

Santamouris, M. (Ed.). (2008). Advances in Building Energy Research- Volume 2. London: Earthscan.

Shen, H., Tan, H., \& Tzempelikos, A. (2011). The effect of reflective coatings on building surface temperatures, indoor environment and energy consumption - An experimental study. Energy \& Buildings, 43, 573-580. http://doi.org/10.1016/j. enbuild.2010.10.024

Suehrcke, H., Peterson, E. L., \& Selby, N. (2008). Effect of roof solar reflectance on the building heat gain in a hot climate. Energy and Buildings, 40, 2224-2235. http:// doi.org/10.1016/j.enbuild.2008.06.015

Synnefa, A., Santamouris, M., \& Akbari, H. (2007). Estimating the effect of using cool coatings on energy loads and thermal comfort in residential buildings in various climatic conditions. Energy and Buildings, 39, 1167-1174. http://doi.org/10.1016/j. enbuild.2007.01.004

Synnefa, A., Santamouris, M., \& Livada, I. (2006). A study of the thermal performance of reflective coatings for the urban environment. Solar Energy, 80, 968-981. http:// doi.org/10.1016/j.solener.2005.08.005

U.S. Environmental Protection Agency. (2008). Cool Roofs. In Reducing Urban Heat Islands: Compendium of Strategies. Recuperado de https://www.epa.gov/heatislands/heat-island-compendium

Uemoto, K. L., Sato, N. M. N., \& John, V. M. (2010). Estimating thermal performance of cool colored paints. Energy and Buildings, 42, 17-22. http://doi.org/10.1016/j. enbuild.2009.07.026

Vecchia, F., Givoni, B., \& Silva, A. (2001). Analyzing thermal performance of occupied houses in Descalvado, Brazil. In Proccedings of 18th PLEA Conference (pp. 1-7). Florianopolis, Brazil. Recuperado de http://www.eesc.sc.usp.br/shs/ attachments/121_ANALYZING_THERMAL_PERFORMANCE.pdf

Yew, M. C., Sulong, N. H. R., Chong, W. T., Poh, S. C., Ang, B. C., \& Tan, K. H. (2013). Integration of thermal insulation coating and moving-air-cavity in a cool roof system for attic temperature reduction. Energy Conversion and Management, 75, 241-248. http://doi.org/10.1016/j.enconman.2013.06.024

Zingre, K. T., Wan, M. P., Tong, S., Li, H., Chang, V. W. C., Wong, S. K., ... Leng Lee, I. Y. (2015). Modeling of cool roof heat transfer in tropical climate. Renewable Energy, 75, 210-223. http://doi.org/10.1016/j.renene.2014.09.045 